\title{
Content-Based Image Retrieval Based on Texture and Color Combinations Using Tamura Texture Features and Gabor Texture Methods
}

\author{
Bahtiar Imran \\ Sekolah Tinggi Manajemen Informatika Komputer Mataram (STMIK Mataram), Mataram, Indonesia
}

Email address:

bahtiarimranlombok@gmail.com

To cite this article:

Bahtiar Imran. Content-Based Image Retrieval Based on Texture and Color Combinations Using Tamura Texture Features and Gabor Texture Methods. American Journal of Neural Networks and Applications. Vol. 5, No. 1, 2019, pp. 23-27. doi: 10.11648/j.ajnna.20190501.14

Received: April 22, 2019; Accepted: June 24, 2019; Published: July 4, 2019

\begin{abstract}
As the development of data storage technology from various sources of information then increasingly cause problems in the search and processing, of course with the existence of this problem will be feared can cause big losses. Various existing search techniques have not been able to provide clear results between query testing and training. To overcome this problem required a Content-Based Image Retrieval (CBIR) approach which is a technique for content-based image search. In this study using texture and color information from image training to present the results both in query testing and database training using Tamura texture method features Gabor texture features. Before displaying the query results first the image testing in extracts using Tamura texture features and Gabor texture features to get the feature values used for image testing and then matching it with the value of features available in the training database. The application used in this research is an application from LIRE and database image that used is database from image. orig. the results obtained in this research is, the application of texture Tamura method features and Gabor texture features based on the features and colors can provide significant results between image testing and image training.
\end{abstract}

Keywords: CBIR, Feature, Tamura Texture Feature, Texture, Matching

\section{Introduction}

Information technology increases so fast in some various aspect such as government, factory, school, hospital, and others [1]. Content-based image search is an application that uses image processing to look for similar, similar images against large databases [2], take the picture the problem is finding large amounts of digital images database for a scientific overview of CBIR field [3]. Content-Based Image Retrieval (CBIR) is a method in which various visual contents (called as features) have been considered to search and retrieve images from the large scale of image databases [4]. The basic principle of this CBIR technique is the use of image analysis algorithms to automatically extract a number of image attributes at a time on the image database. This attribute can include numeric values for colors, textures, and shapes. Due to the complexity of image information, the user cannot expect to get a certain match between the query and the image to be displayed back from the database [6]. CBIR is a technique for image search by content. Various search methods have not been able to provide significant results associated with image search. There are some obstacles encountered for the current search method that are too large file size, file name inequality, and file location.

Textual shooting depends on the attached image, label or metadata with each digitally stored image. Then the database query is used to retrieve the image containing the query keyword from the user query input. However, in order to use image metadata, all digital images must be described with metadata [7]. Characteristics of the resulting image can be shape, color, texture and other features [8]. The texture is one feature that has important information from each of the values that exist in an image to describe a state and its surface structure. To obtain information from a texture it needs to do feature extraction, one of the methods that can be used for feature extraction is Tamura feature extraction method.

Many previous studies have conducted research on CBIR with various methods, such as astute, hariadi and full moon 
(2009) study on CBIR based on shape feature using gradient vector flow snake method to get CBIR conclusion by using GVF snake can be initialized inside or outside the image so that more accurate accuracy in recognizing the shape features in accordance with the desired.

Furthermore, in the study of halim, hardy, goddess and space (2013) on the application of image retrieval using a combination of color moment method and Gabor texture concluded the combination of color moment region and Gabor texture can display more relevant image search results indicated by more precision and recall value high compared to other combinations.

In this research, Tamura feature extraction and Gabor texture feature are used for extraction of an image both image testing and image training in the database, then matching it to get the output of each image in testing.

\section{Literature Review}

\subsection{Content-Based Image Retrieval}

Content-Based Image Retrieval System (CBIR) is an image retrieval technique that has similar characteristics or content of a set of images [6]. then index those features by using some appropriate structure and then efficiently provide the result to the users [12]. In CBIR, visual features such as color and texture are extracted to characterize the image. CBIR includes many methods from the field of image processing and computer vision and is considered part of that field. In CBIR, visual content is extracted and explained by a multidimensional feature vector. To take a picture, the user provides a search system with a sample image. Tasks performed by CBIR can be classified into pre-processing and feature extraction phases. In the pre-processing stage, noise removal and improvement of some features of the relevant objects to display query input results. Image segmentation is also performed for separate objects from the background image [9]. Efficient image searching, browsing and retrieval tools are required by users from various domains, including remote sensing, fashion, crime prevention, publishing, medicine, architecture, etc [15].

\subsection{Color}

Color is an important and most visible feature that humans receive or feel when first seeing a picture. The number of color space systems built to represent colors such as RGB and HSV. RGB is a color model based on the concept of adding strong primary light that is Red, Green, and Blue. If in a totally dark space, no lightwave signal is absorbed by the eye or RGB $(0,0,0)[8]$. In RGB color, color is represented by triplets $(R, G, B)$, where $R$ gives the intensity of the red component, $\mathrm{G}$ gives the intensity of the green component and $\mathrm{B}$ gives the intensity of the blue component. Basically, the color model is the specification of the coordinate system and the inner space, where each color is represented in a single point. He is the dominant wavelength in the light. This is the term for pure spectrum colors. Hue is expressed from $0^{\circ}$ to $360^{\circ}$. It is red (starting at $0^{\circ}$ ), yellow (starting at $60^{\circ}$ ), green (starting at $120^{\circ}$ ), cyan (starting in $180 \mathrm{~s}$ ), blue (starting at $240^{\circ}$ ) and magenta (starting at $300^{\circ}$ ). Eventually, all colors can be mixed with the three basic colors known as the introduction. Saturation represents the dominance of color hue. It is defined as the degree of color purity. Values describe the brightness or intensity of the color. It can also be defined as a relatively light or dark color [9]. Color is also an intuitive feature and plays an important role in image matching [13].

\subsection{Texture}

Image textures are defined as images of natural textured surfaces and artificially created polavisuals. The texture is the regularity of certain patterns formed from the arrangement of the pixels in the image. Textures (textures) indicate the nature or characteristics possessed by a large area so that naturally these traits can be repeated in the area. Texture analysis works by observing the pattern of neighboring values between pixels in the spatial domain. In addition, textures typically describe the intrinsic characteristics of the image by measuring roughness, granularity, and regularity of the structural arrangement of pixels. The textural aspect of an image can be utilized as the basis of segmentation, classification, or image interpretation [8]. The texture descriptors provide measure of properties such as smoothness, coarseness and regularity. Statistical approaches yield characterization of texture as a smooth, coarse, grainy and so on. Texture determination is ideally suited for medical image retrievals [14].

\subsection{Tamura Texture Feature}

Tamura features texture approaches by designing texture features that fit the human visual perception. Tamura texture features the six features of texture (coarseness, contrast, directionality, line-likeness, regularity, and roughness) and compares it with psychological measurements. Coarseness: Roughness has a direct relationship with the scale and repetition rate of this feature in Tamura feature extraction is the most basic texture feature.

$$
\begin{gathered}
A_{k}(x, y)=\sum_{i=x-2^{k-1}}^{x+2^{k-1}} \sum_{j=y-2^{k-1}}^{y+2^{k-1}-1} \frac{f(i, j)}{2^{2 k}} \\
E_{k, h}(x, y)=\left|A_{k}\left(x+2^{k-1}, y\right)-A_{k}\left(x-2^{k-1}, y\right)\right|
\end{gathered}
$$

Contrast: aims to capture the dynamic range of the gray level in the image, along with the black and white distribution polarization. The first is measured using the standard deviation of the gray level and the two $\alpha 4$ kurtoses. The size of the contrast is defined as.

$$
F_{\text {con }}=\frac{\sigma}{\left(\alpha_{4}\right)^{n}} \text { where } \alpha_{4}=\frac{\mu_{4}}{\sigma^{4}}
$$

Directionality: is a global property in an area. The described feature does not aim to distinguish between different orientations or patterns, but the total step of the level of directionality. At each angle pixel and the magnitude is calculated. A histogram, $\mathrm{Hd}$, probability edge is then 
constructed by calculating all the points of magnitude greater than the threshold and quantizing by the edges of the edge. The histogram will reflect the level of directionality [8]-[9].

\subsection{Gabor Texture Feature}

The Gabor filter is constructed by modulating a sine or cosine wave with a Gaussian, thus providing the optimally combined localization in space and frequency. The modulation of sine waves with gaussian provides localization in space but eliminates localization in frequency. The decomposition of a signal is performed with a pair of Gabor quadrature filters, with real sections being specified by a cosmic wave modulated with Gaussian, and imaginary parts specified by a Gaussian modulated sine wave. Real and imaginary filters are also known as the symmetric and old symmetric components [8]. Gabor has a strong dependence on a number of parameters that affect the performance of texture characterization [10]. Gabor filters are found to perform better than wavelet transform and other multiresolution approaches in representing textures and retrieving images due to its multiple orientation approach [11] .

\section{Research Method}

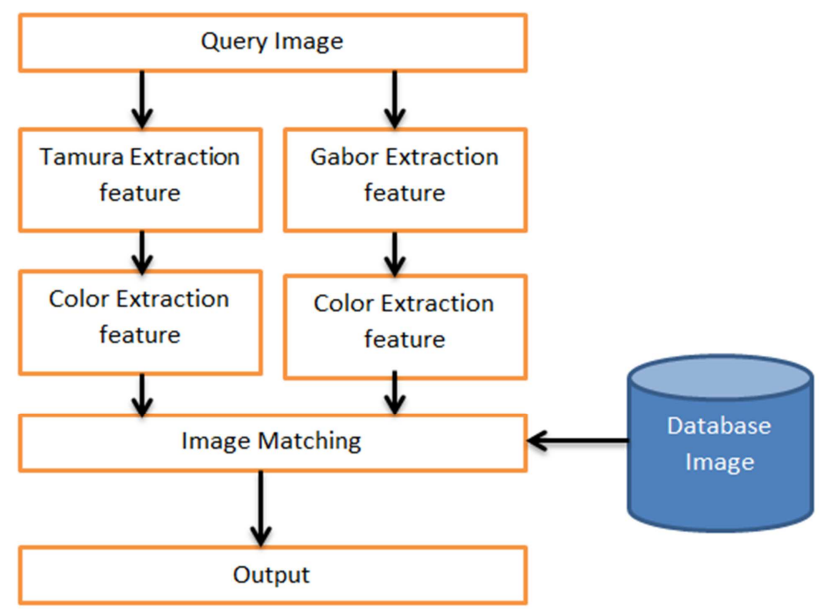

Figure 1. Diagram Process.

Research is done by using an image database of image. orig with the following characteristics:

a) The observed picture data amounted to 999 pieces.

b) Each image is jpg format

c) The resolution of the searched and queried images is $384 \times 256$ pixels and $256 \times 384$ pixels

In this research, there are several processes that will be done from the query image testing, texture analysis, color analysis, until the results have been matching with query testing.

The processes are:

a) Enter the query image testing.

b) Extraction feature texture of image query using Tamura feature extraction

c) Extraction feature color query image using Tamura feature Extraction feature of the texture of image query using Gabor texture feature

d) Extraction feature color query image using Gabor texture feature.

e) Matching image on database

Show matching results.

Here is a picture or model flowchart of the process that will be done in this research:

\section{Results and Discussion}

The working procedure of the developed image search application consists of:

a) Training process. The training process is conducted with the aim to provide information to the system about the characteristics of the image.

b) Testing process. The testing process is a process that aims to perform a similar or similar image search on the database.

The application administrator must enter the folder location information where the training dataset is stored. In this study, the number of images contained in the database consists of 999 taken on the image. orig database with various categories.

Applications can display search results based on the choice of the algorithm used. The search results are displayed in the form of images that have been matched between the query and the existing image database training. The output results are based on the algorithm used. Prior to the matching stage, first image query testing in texture feature extraction and color is then matched to the existing image in the training database. Figure 2 shows an example of the result of an image search.

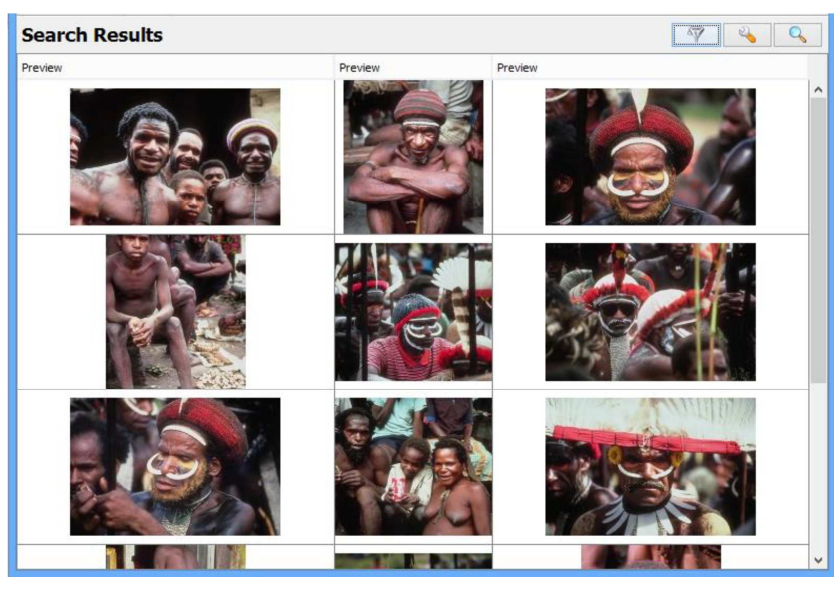

Figure 2. Search Result.

In Figure 2 shows the search results using Tamura texture features, before the image query is done first image testing is prepared, image testing used is image 401.jpg, Figure 3 shows the image testing used. This image testing will be equated at the time of testing. The reason for using image query testing is the same between Tamura texture features and Gabor texture features is to find which method produces the most relevant results. 


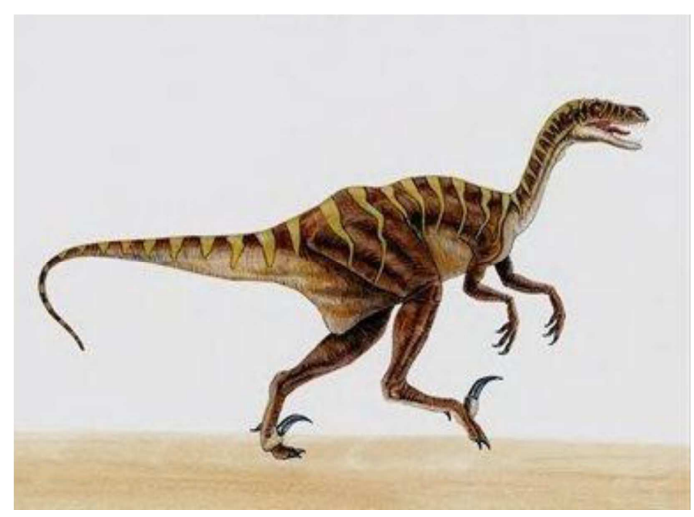

Figure 3. Image Testing Used.

After the image testing is ready, then enter the image testing in the search menu. For the results using Tamura method, the texture feature can be seen in figure 4 .

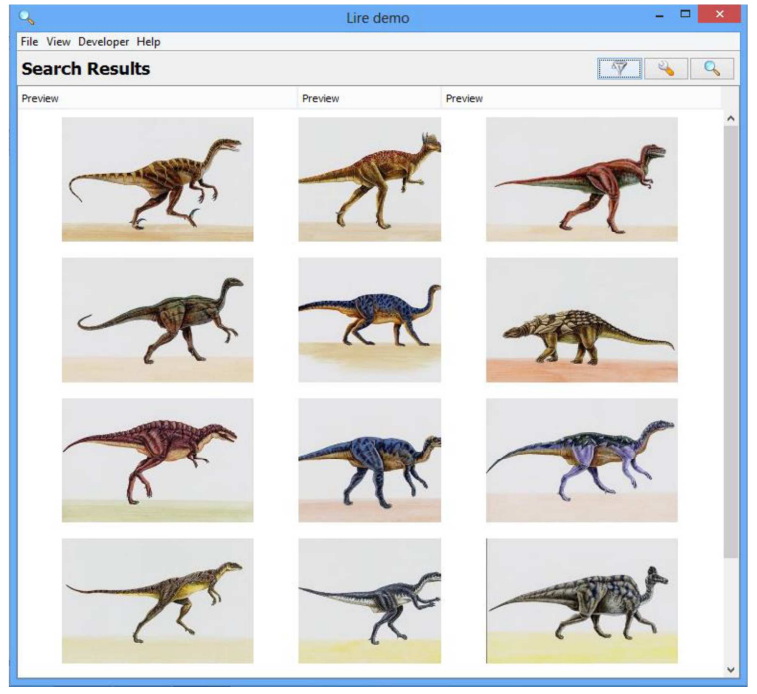

Figure 4. Search Using Tamura Texture Features.

Figure 5 shows the search results using Gabor texture features. Image testing is used using the same image testing as Tamura texture feature ie image 401.jpg.

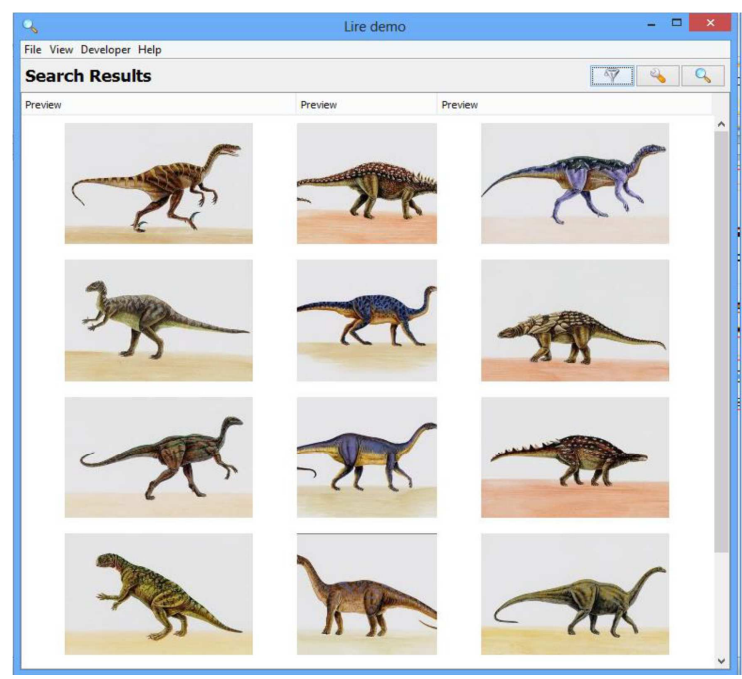

Figure 5. Search Using Gabor Texture Features.

\section{Conclusion}

The conclusion of this research is that the use of texture Tamura feature and Gabor texture feature based on the combination of texture and color has resulted significantly between the image testing in input an with the resulting image, this is evident from the example image testing 401. jpg used can display output is almost similar to image testing.

For the effect of noise on image testing and image, training is very influential on the results obtained. For further research, it is expected that the use of filters before the testing and training stage is done the first image that will be used in the filter first. Then it is also expected to conclude between the image testing and the output can use Euclidean distance, cosine similarity, and others.

\section{References}

[1] Suharjito, et al., "Family Relationship Identification by Using Extract Feature of Gray Level Co-occurrence Matrix (GLCM) Based on Parents and Children Fingerprint," International Journal of Electrical and Computer Engineering (IJECE), vol. 7, October 2017, pp. 2738-2745.

[2] M. D. Agaputra, et al., "Pencarian Citra Digital Berbasiskan Konten dengan Ekstraksi Fitur HSV, ACD, dan GLCM, vol. 8, pp. 8-13.

[3] B. M. S and Naik. M. B., "Content Based Image Retrieval Using Color and Texture Content," International Journal of Computer Trends and Technology (IJCTT), vol. 48, June 2017, pp. 78-84.

[4] N. V. M. K. Raja and K. S. Banu, "Content Bases Image Search And Retrieval Using Indexing By KMeans Clustering Technique," International Journal of Advanced Research in Computer and Communication Engineering, vol. 2, May 2013, pp. 2181-2189.

[5] R. B and K. R. Chandran., "Content Based Medical Image Retrieval with Texture Content Using Gray Level Cooccurrence Matrix and K-Means Clustering Algorithms", Journal of Computer Science, Vol. 8, 2012, pp. 1070-1076.

[6] I. Hastuti, et al., "Content Based Image Retrieval Berdasarkan Fitur Bentuk Menggunakan Metode Gradient Vector Flow," Seminar Nasional Informatika UPN Veteran Yogyakarta, 2009, pp. 140-145.

[7] T. Mehyar and O. J. Atoum., "An Enhancement on ContentBased Image Retrieval using Color and Texture Features," Journal of Emerging Trends in Computing and Information Sciences, vol. 3, pp. 488-496.

[8] A. Halim, et al., "Aplikasi Image Retrieval Menggunakan Kombinasi Metode Color Moment dan Gabor Texture, "JSM STMIK Mikroskil, vol. 14, October 2013, pp. 109-117.

[9] S. V. B and J. M. David., "Content Based Image Retrieval: Classification Using Neural Networks," The International Journal of Multimedia \& Its Applications (IJMA), vol. 6, October 2014, pp. 31-44.

[10] Wicaksono. Y, et al., "Color and Texture Feature Extraction Using Gabor Filter - Local Binary Patterns for Image Segmentation with Fuzzy C-Means," Journal of Intelligent Systems, Vol. 1, No. 1, February 2015. 
[11] Jain. N and Salankar. S. S., "Content Based Image Retrieval Using Gabor Texture Feature and Color Histogram", International Journal of Enhanced Research in Science Technology \& Engineering, Vol. 3 Issue 9, Sept.-2014, pp: 97102.

[12] Kumari. B., "CBIR Systems: Results of Feature Extraction with Color Feature Comparison with Standard Database", International Journal of Innovative Research in Computer and Communication Engineering, Vol. 5, Issue 11, November 2017.

[13] Singha. M., "Content Based Image Retrieval using Color and Texture," Signal \& Image Processing: An International
Journal (SIPIJ), Vol. 3, No. 1, February 2012.

[14] Thawari. P. B. and Janwe. N. J., "CBIR BASED ON COLOR AND TEXTURE", International Journal of Information Technology and Knowledge Management, Vol. 4, No. 1, pp. 129-132.

[15] Yadav. A. K. r, et al., "Survey on Content-based Image Retrieval and Texture Analysis with Applications", International Journal of Signal Processing, Image Processing and Pattern Recognition, Vol. 7, No. 6 (2014), pp. 41-50. 